\title{
Muon Identification and Isolation efficiencies on Run II data with the CMS experiment
}

\author{
Pedro J. Fernández Manteca ${ }^{1}$ \\ Instituto de Física de Cantabria (Universidad de Cantabria - CSIC) \\ Edificio Juan Jordá. Avenida de los castros s/n, Santander, Spain \\ E-mail: pedro.fernandez.manteca@cern.ch
}

\begin{abstract}
The performance of muon identification and isolation efficiencies in CMS has been studied on data collected in proton-proton collisions at $13 \mathrm{TeV}$ at the LHC on the full 2016 dataset. The efficiencies have been computed with the tag-and-probe method, in different periods of data taking. Results obtained using data are compared with Monte-Carlo predictions.
\end{abstract}

Sixth Annual Conference on Large Hadron Collider Physics (LHCP2018)

4-9 June 2018

Bologna, Italy

\section{${ }^{1}$ Speaker}




\section{Introduction: the Tag-and-Probe method}

The identification (ID) and isolation (ISO) muon efficiencies in CMS experiment are estimated by using the Tag-and-Probe method [1], whose performance is described in this section.

The Tag-and-Probe method is a very common procedure for measuring efficiencies in High Enery Physics. First, a well known resonance is considered, in this case two muons that come from the $\mathrm{Z}$ boson peak are selected. Then, one muon is named as "Tag" and the other as "Probe" for both data and simulation.

The "Tag" muons pass very tight requirements of ID and ISO, while the "Probe" muons correspond to compatible tracks with the $\mathrm{Z}$ boson resonance. The aim is to measure a certain ID or ISO efficiency over the "Probe" muons.

Since the selected events may not come from the resonance, there could be a bias in the efficiency measurement. To avoid this, a simultaneous fir to the signal ( $Z$ peak) and the background is performed (as shown in Figure 1), and in order to be able to fit the background properly, a wide mass range (for instance $70-130 \mathrm{GeV}$ ) has to be considered.
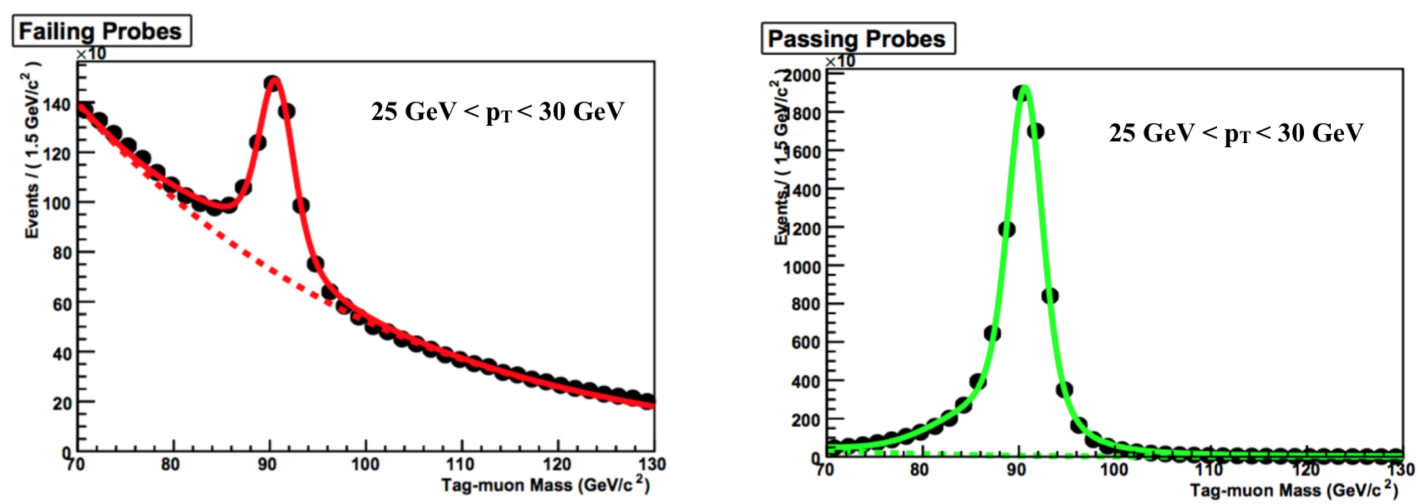

Figure 1: Failing (left plot) and Passing probes (right plot) fits examples for Tight ID working point, $25<\mathrm{p}_{\mathrm{T}}<30 \mathrm{GeV}$ bin.

At the end of this proccess, the total probe sample is split considering whether the mons pass (Passing Probes) or do not mass (Failing Probes) the ID or ISO working point that we want to measure. The fit is done independently for both the Passing Probes and the Failling Probes, and finally the efficiency is computed as:

$$
\varepsilon=\frac{\text { passing probes }}{\text { passing probes }+ \text { failling probes }}
$$

One thing to remark is that if we want to measure the efficiency as function of one kinematic variable, this proccess will have to be repeated for each variable bin. 


\section{Data / Monte-Carlo samples, fitting parameters, and event selection}

The details on the Data and Monte-Carlo samples used in this study, on the fitting parameters that are set for the Tag-and-Probe method, and on the chosen event selection are described in this section.

The efficiency is computed for several working points based on quality requirements on the muon ID and ISO (more details in [1]). In particular, the two most used working points in CMS are the Tight muon ID, and the Tight muon ISO:

- Tight muon ID aims at suppressing muons from decay in flight and from hadronic punchthrough.

- Tight muon ISO removes muons with a relative isolation above 0.15 . The relative muon isolation is defined as the sum of the energy relative to the muon transverse momentum $\left(\mathrm{p}_{\mathrm{T}}\right)$ inside a cone of radius $\Delta \mathrm{R}=\left(\Delta \varphi^{2}+\Delta \eta^{2}\right)^{1 / 2}$ centered on the muon direction, where $\varphi$ corresponds to the azimuthal angle and $\eta$ to the pseudorapidity.

The systematic uncertainty on the measurements can be evaluated by varying the "Tag" muon definition, the fit functions, the number of mass bins, or the mass range where the fits are performed. The impact of the variations on top of the efficiencies is in most of the cases less than $0.5 \%$.

\subsection{Data and Monte-Carlo samples}

For data, the full 2016 dataset is considered, corresponding to CMS collision data at 13 $\mathrm{TeV}$ and 25 ns bunch spacing, with a total luminosity of $36 \mathrm{fb}^{-1}$. Concerting the Monte-Carlo simulation, a Drell-Yan plus Jets sample generated with MadGraph5_aMC@NLO [2] has been used, for which an event re-weighting has been applied in order to match the pileup distribution in data.

\subsection{Fitting parameters}

The considered mass window is $70-130 \mathrm{GeV}$ for ID efficiencies and $77-130 \mathrm{GeV}$ for ISO efficiencies. The reason why the ID one is wider is because the amount of background is much larger for ID efficiencies than for ISO efficiencies, and then fitting the background properly is so important in the ID case.

About the fitting functions, for ID measurements as function of the transverse muon momentum the signal is fitted by the sum of two Voigtians, while the background is fitted by a product of an exponential function and an error function. In other cases the background is simply fitted by an exponential function.

\subsection{Event selection}

"Tag” muons are required to pass the Tight muon ID, a single muon trigger that includes a $\mathrm{p}_{\mathrm{T}}>24 \mathrm{GeV}$ cut and a relative isolation requirement below 0.40 , a selection on transverse momentum greater than $26 \mathrm{GeV}$, and finally a relative isolation below 0.2 .

For ID efficiencies the "Probe" muons are required to be have $\mathrm{p}_{\mathrm{T}}>20 \mathrm{GeV}$, while for ISO efficiencies they have to pass a certain ID working point and $\mathrm{p}_{\mathrm{T}}>20 \mathrm{GeV}$. 


\section{Results}

The efficiency measurements for Tight muon ID as function of the muon pseudorapidity $\eta$, and for Tight muon ISO as function of the muon p T are shown in Figure 2 (more plots in [2]).

The global efficiencies for all the studied muon working points are registered in Table 1.
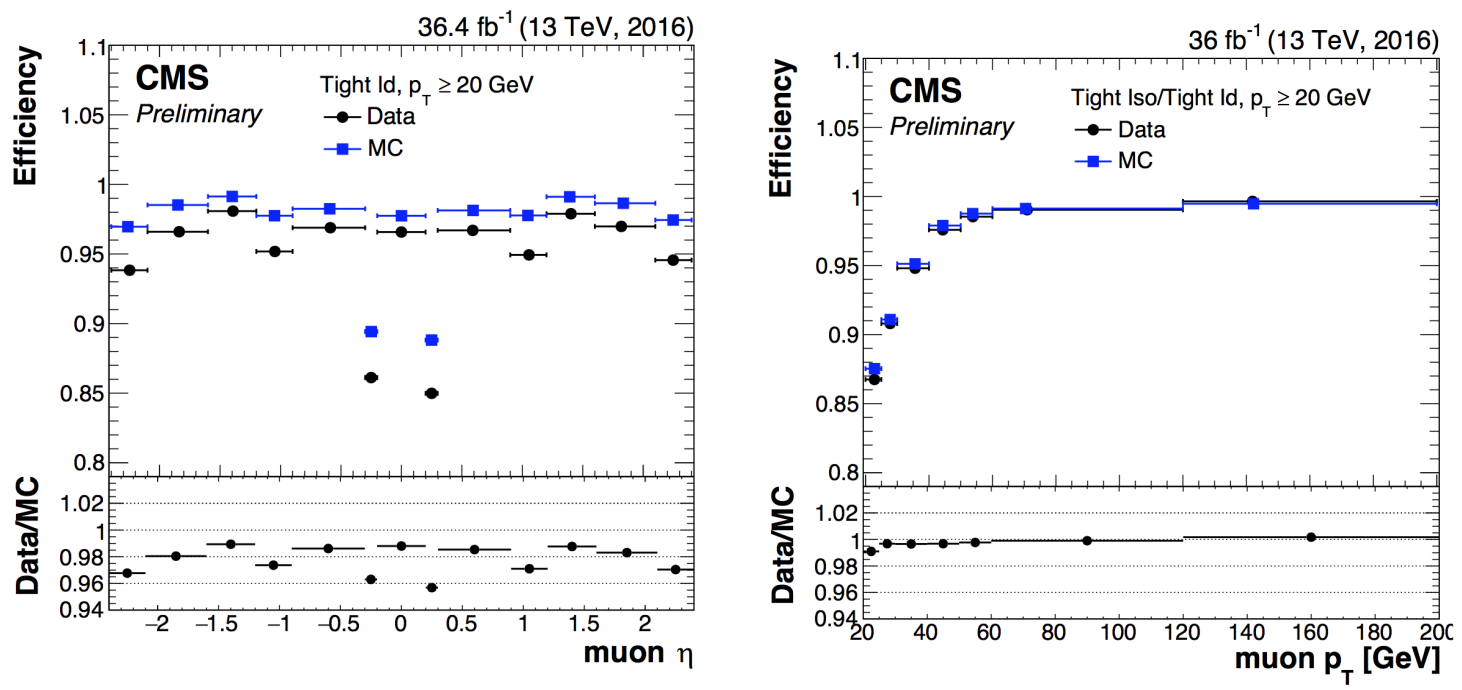

Figure 2: Efficiency measurements for Tight muon ID working point as function of muon $\eta$ on the left, and for Tight muon ISO working point on top of Tight muon working point on the right. Data is plotted in dark and Monte-Carlo simulation in blue. The ratio between data and simulation is placed at the bottom.

\begin{tabular}{|c|c|}
\hline Working Point & Global efficiency \\
\hline Loose ID & $\sim 99 \%$ \\
\hline Medium ID & $\sim 97 \%$ \\
\hline Tight ID & $\sim 96 \%$ \\
\hline High-p T ID & $\sim 96 \%$ \\
\hline Loose ISO / Loose ID & $\sim 99 \%$ \\
\hline Loose ISO / Medium ID & $\sim 99 \%$ \\
\hline Loose ISO / Tight ID & $\sim 99 \%$ \\
\hline Loose Trk ISO / High-p T ID & $\sim 98 \%$ \\
\hline Tlght ISO / Medium ID & $\sim 96 \%$ \\
\hline Tight ISO / Tight ID & $\sim 96 \%$ \\
\hline
\end{tabular}

Table 1: Global efficiency summary for all the studied working points. 


\section{References}

[1] The CMS collaboration, Performance of the CMS muon detector reconstruction with proton-proton collisions at $13 \mathrm{TeV}, 2018$ JINST 13 P06015 [arXiv: 1804 . 04528 ]

[2] J. Alwall, R. Frederix et al, The automated computation of tree-level and next-to-leading order differential cross sections, and their matching to parton shower simulations, JHEP 07 (2014) 079 [arXiv:1405.0301]

[3] The CMS collaboration, Muon Identification and Isolation efficiency on full 2016 dataset, CMSDP-20017/007 